\title{
Salicylanilide /Cyclodextrin Inclusion Complex: Preparation, Characterization and Molecular Docking Studies
}

\author{
K Sivakumar* and M Parameswari \\ Department of Chemistry, Faculty of Science, Tamilnadu, India
}

Received: November 20, 2015; Accepted: November 30, 2015; Published: December 05, 2015

*Corresponding author: Dr. K Sivakumar, Department of Chemistry, Sri Chandrasekharendra Saraswathi Viswa MahaVidyalaya University, Enathur, Kanchipuram - 631 561, Tamilnadu, India. Tel: +91 9842361378; Fax: +91 044 27264285; E-mail: chemshiva@gmail.com

\begin{abstract}
Interaction between Salicylanilide (SA) with $\beta$-Cyclodextrin $(\beta$ CD) has been investigated by UV and fluorescence techniques. The red shift in $\lambda_{\max }$ enhanced absorption and emission in $\beta$-CD medium confirms the $-\mathrm{NH}-\mathrm{CO}-\mathrm{C}_{6} \mathrm{H}_{5}$ aromatic side chain $\mathrm{SA}$ is inserted in the $\beta$-CD cavity. The stoichiometry and binding constant of 1:1 inclusion complex was calculated using the Benesi-Hildebrand plot derived UV and fluorescence studies and the thermodynamic parameters $(\Delta \mathrm{G}, \Delta \mathrm{H}$ and $\Delta \mathrm{S})$ of inclusion process were also determined. The results indicated that the inclusion process was an exergonic and spontaneous process. The complex was investigated by FTIR, XRD, Semi empirical and molecular docking methods. The SA: $\beta-C D$ inclusion complex obtained by molecular docking studies was in good correlation with the results obtained through experimental methods.
\end{abstract}

Keywords: $\beta$-Cyclodextrin; Salicylanilide; Inclusion Complex; Patchdock Server

\section{Introduction}

Salicylanilides (SA) is the amide of salicylic acid and aniline. It is classified as both a salicylamide and an anilide. SA is a group of anthelmintics which exert their action by uncoupling mitochondrial reactions which are critical to electron transport and associated phosphorylation in the metabolic system of the parasite. They are effective against cestodes and trematodes but not nematodes. Some are active against Haemonchus contortus. Derivatives of salicylanilide have a variety of pharmacological uses. Chlorinated derivatives including niclosamide, oxyclozanide and rafoxanide are used asanthelmintics, especially as flukicides. Brominated derivatives including dibromsalan, metabromsalan and tribromsalan are used as disinfectants with antibacterial and antifungal activities.

CycloDextrins (CDs) are cyclic oligosaccharides obtained from enzymatic hydrolysis of starch. The $\beta$-CD is the most abundant natural oligomers and corresponds to the association of seven glucose units with cavity, which exhibits a hydrophobic character whereas the exterior is strongly hydrophilic. Their ability to form host-guest complexes has led to the use of CDs in a number of fields [1, 2]. CDs have been used in the pharmaceutical industry, as solubilizers, diluents and as tablet ingredients which improve the chemical stability, solubility, bioavailability and pharmacokinetic properties of drugs. In this paper, we report the photo physical and computational studies on the complexation SA and $\beta$-CD at different conditions. In addition to the UV and Fluorescence studies, we have utilized the complexation behavior of SA for the stoichiometry and binding constant of SA: $\beta$-CD inclusion complex. Further it was also supported by XRD, semiemprical method and molecular docking studies.

\section{Materials and Methods}

\section{Materials}

Salicylanilide and $\beta$-CD were obtained from Aldrich, Hi Media Laboratories and used without further purification. Triply distilled water was used to prepare all solutions and spectro grade solvents were used. Solutions in the $\mathrm{pH} 7$ were prepared by adding the appropriate amount of $\mathrm{NaOH}$ and $\mathrm{H}_{3} \mathrm{PO}_{4}$. The concentration of $\beta$-CD was varied from zero to $1.2 \times 10^{-3} \mathrm{~mol} \mathrm{dm}^{-3}$. From the stock solution 2, 4, 6, 8, 10 and $12 \times 10^{-3} \mathrm{~mol} \mathrm{dm}^{-3}$ of $\beta$-CD were prepared using $\mathrm{pH} \sim 7$ buffers. The concentrations of the solutions were of the order $10^{-4} \mathrm{~mol} \mathrm{dm}^{-3}$. All experiments were carried out at $30^{\circ} \mathrm{C}$. The solid inclusion complex was also prepared by coprecipitation method.

\section{Methods}

The $\mathrm{pH}$ values were measured using Elico $\mathrm{pH}$ meter LI120. The UV spectra were recorded with Specord 200+ spectrophotometer, Germany. The Fluorescence spectra were recorded using Spectro fluorometer, Perkin Elmer, USA. The IR spectra of all samples were recorded using Alpha-T FTIR Spectrometer (Bruker optics) equipped $\mathrm{KBr}$ by using a clean glass pestle and mortar. Powder X-ray diffraction spectra were taken by XPert PRO PANalytical diffractometer. The most probable structure of the SA: $\beta$-CD inclusion complex was determined by molecular docking studies using PatchDock server [3]. 


\section{Results and Discussion}

\section{Host-Guest Interaction of SA with $\beta$-CD}

The absorption spectral data of SA in different concentrations of $\beta$-CD recorded in $\mathrm{pH} \sim 7$ are compiled in Table 1. In SA upon increasing the concentration of $\beta$-CD a slight red shift is observed in the absorption maxima in the LW and SW absorption bands. No clear isosbestic point is observed in absorption spectrum. The absorption spectra show only very slight change in absorption maxima even in the presence of highest concentration of $\beta$-CD used $\left(12 \times 10^{-3} \mathrm{M}\right)$ in $\mathrm{pH} \sim 7$. This behavior has been attributed to the enhanced dissolution of SA molecules through the hydrophobic interaction between guest molecule (SA) and nonpolar cavity of $\beta$-CD [4-6] as reported by others also [7, 8]. Since, this indicates the formation of 1:1, host-guest inclusion complex of SA: $\beta$-CD.

The binding constant for the formation of SA: $\beta$-CD complex has been determined by analyzing the changes in the intensity of absorption maxima with the $\beta$-CD concentration. In the case of inclusion complex formed between SA and $\beta-C D$, the equilibrium can be written as,

$$
\text { Salicylanilide }+\beta-C D \stackrel{k}{\rightleftharpoons} \text { Salicylanilide.... } \beta-C D
$$

The binding constant ' $\mathrm{K}$ ' and stoichiometric ratios of the inclusion complex of SA can be determined according to the Benesi-Hildebrand [9] relation assuming the formation of a 1:1 host-guest complex.

$$
\frac{1}{\mathrm{~A}-\mathrm{A}_{0}}=\frac{1}{\Delta \varepsilon}+\frac{1}{K[\text { Salicylanilide }]_{0} \Delta \varepsilon[\beta-\mathrm{CD}]_{0}}
$$

Where, $\mathrm{A}$ and $\mathrm{A}_{0}$ is the difference between the absorbance of SA in the presence and absence of $\beta-C D, \Delta \varepsilon$ is the difference between the molar absorption coefficient of $S A$ and the inclusion complex, $[\mathrm{SA}]_{0}$ and $[\beta-\mathrm{CD}]_{0}$ are the initial concentration of $\mathrm{SA}$ and $\beta$-CD respectively. The plot of $1 / A-A_{0}$ verses $1 /$ [ $\beta$-CD] for SA in $\mathrm{pH} \sim 7$. For $\mathrm{pH} \sim 7$ solutions, a good linear correlation was obtained, confirming the formation of a 1:1 inclusion complex. From the intercept and slope values of this plot, the binding constant ' $\mathrm{K}$ ' was evaluated. The ' $\mathrm{K}$ ' value for SA in neutral condition (258.5 $\mathrm{M}^{-1}$ at $\mathrm{pH}$ 7) at $303 \mathrm{~K}$

The effect of $\beta$-CD on the fluorescence spectra of SA (Table 1 ) is different from absorption spectra and more pronounced than the relative effect on the absorption spectra. In SA, there is no significant change is observed in emission maxima $(\sim 528$ $\mathrm{nm})$ at $\mathrm{pH} \sim 7$. The emission intensity of $\mathrm{SA}$ in $\mathrm{pH} \sim 7$ is increases when the $\beta$-CD concentration is increased (Figure 1), whereas the intensity is increased. Figure 1 shows the Benesi-Hildebrand plot of observed changes in the fluorescence intensity with increasing concentration of $\beta$-CD. It is seen from this plot that the emission

intensity of SA initially increases with $\beta$-CD concentration and then saturates to a limiting value at $0.012 \mathrm{M} \beta$-CD, indicating the maximum inclusion of SA molecule in the $\beta$-CD cavity. The

binding constant for the formation of complex has been

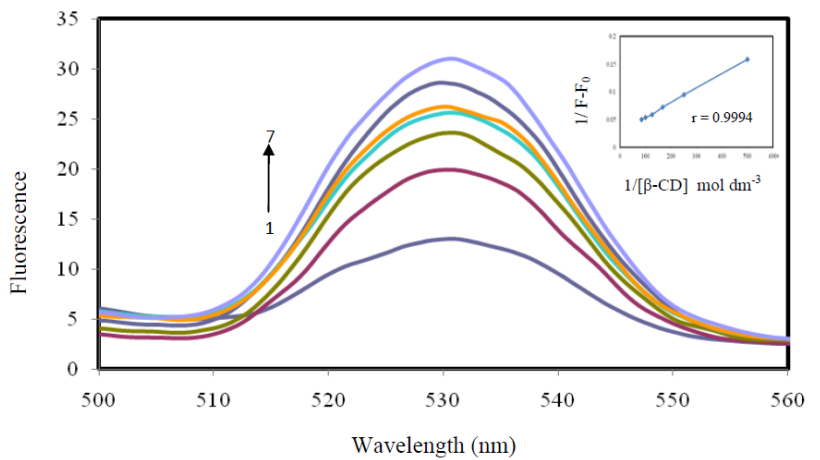

Figure 1: The Fluorescence spectra of Salicylanilide $(\mathrm{pH} \sim 7)$ in different $\beta$-CD concentrations ( $\mathrm{mol} \mathrm{dm}^{-3}$ ): (1) $0.0 \mathrm{M},(2) 0.002 \mathrm{M}$, (3) $0.004 \mathrm{M},(4)$ $0.006 \mathrm{M}$, (5) 0.008 M, (6) 0.010M and (7) $0.012 \mathrm{M}$. (Inside- Benesi-Hildebrand plot of $1 / \mathrm{F}-F_{0}$ vs. $1 /[\beta-\mathrm{CD}]$ for Salicylanilide in $\mathrm{pH} \sim 7$ solution).

determined by analyzing the changes in the intensity of emission maxima with the $\beta$-CD concentration using the BenesiHildebrand[9] relation assuming the formation of a 1:1 host guest complex.

$$
\frac{1}{\mathrm{I}-\mathrm{I}_{0}}=\frac{1}{\mathrm{I}^{\prime}-\mathrm{I}_{0}}+\frac{1}{K\left[\mathrm{I}^{\prime}-\mathrm{I}_{0}\right][\beta-\mathrm{CD}]_{0}}
$$

Where, $[\beta-C D]_{0}$ represents the initial concentration of $\beta-C D$, " $\mathrm{I}_{0}$ " and "I" are the fluorescence intensities in the absence and presence $\beta$-CD respectively, and I' is the limiting intensity of florescence. The ' $\mathrm{K}$ ' value was estimated from the slope and intercept of the Benesi-Hildebrand plot which shows a good linear correlation supporting the assumption of $1: 1$, SA: $\beta$-CD inclusion complex. The binding constant ' $\mathrm{K}$ ' value is SA evaluated as $375.14 \mathrm{M}^{-1}$.

\section{The thermodynamics of inclusion process}

The thermodynamic parameters $\Delta \mathrm{G}$ for the binding of guest molecule to $\beta$-CD cavity can be calculated from the binding constant ' $\mathrm{K}$ ' by using the following equation

$$
\Delta \mathrm{G}=-\mathrm{RT} \ln K
$$

The thermodynamic parameters $\Delta \mathrm{G}$ for the binding of guest molecules (SA) to $\beta$-CD cavity are given in Table 1 . The negative value of $\Delta G$ suggests that the inclusion process proceeded spontaneously at 303K. Considering the above discussions, the possible inclusion mechanism is proposed. Naturally, The inclusion complex formation of SA with $\beta$-CD, both guest molecules with the $-\mathrm{NH}-\mathrm{CO}-\mathrm{C}_{6} \mathrm{H}_{5}$ aromatic side chain and benzene ring of SA inserted in the $\beta$-CD cavity as shown in Scheme 1.

\section{Characterization of SA: $\beta$-CD complex}

The infrared (FTIR) spectra of wave number from 4000 to $400 \mathrm{~cm}^{-1}$ of salicylanilide, $\beta$-CD and the solid inclusion complex of Salicylanilide with $\beta$-CD were registered by FTIR spectrometer. The host molecule $(\beta-C D)$ reacts with guest molecule (SA) to form host-guest solid complex (SA: $\beta$-CD). The solid complex formation can be confirmed by FTIR spectroscopy because, the bands resulting from the included part of the guest molecule are 
Table 1: Absorption and fluorescence maxima ( $\mathrm{nm})$ of SA (0.001 M) at different concentrations of $\beta$-CD in $\mathrm{pH} \sim 7$ solutions.

\begin{tabular}{|c|c|c|c|c|c|}
\hline \multirow[t]{8}{*}{ S. No. } & $\begin{array}{c}\text { Concentration of } \\
\beta \text {-Cyclodextrin (M) }\end{array}$ & $\lambda_{\max (\mathrm{nm})}$ & $\log \varepsilon$ & $\lambda_{\mathrm{flu}(\mathrm{nm})}$ & Flu. Inten \\
\hline & $\begin{array}{c}0 \\
\text { Without } \beta-C D\end{array}$ & 268 & 3.55 & 528 & 13.403 \\
\hline & 0.002 & 262 & 3.61 & 529.5 & 19.702 \\
\hline & 0.004 & 262 & 3.63 & 529.5 & 23.958 \\
\hline & 0.006 & 262 & 3.63 & 529.5 & 24.18 \\
\hline & 0.008 & 264 & 3.65 & 529.5 & 24.6 \\
\hline & 0.01 & 264 & 3.66 & 530 & 31.821 \\
\hline & 0.012 & 268 & 3.7 & 531 & 33.29 \\
\hline \multicolumn{2}{|c|}{ Binding constant $\left(\mathrm{M}^{-1)}\right.$} & \multicolumn{2}{|c|}{258.5} & \multicolumn{2}{|c|}{375.14} \\
\hline \multicolumn{2}{|c|}{$\Delta G\left(\mathrm{~kJ} \mathrm{~mol}^{-1}\right)$} & \multicolumn{2}{|c|}{-13.99} & \multicolumn{2}{|c|}{-14.93} \\
\hline
\end{tabular}

\section{Scheme 1:}

\begin{tabular}{|c|c|c|c|c|}
\hline \multirow{2}{*}{ Guest } & \multicolumn{2}{|c|}{ Bond distance in ${ }^{\circ} \mathrm{A}$} & Orientation & inclusion \\
\hline \multirow{2}{*}{$\mathrm{SA}$} & $\mathrm{H}_{17}-\mathrm{H}_{25}$ & 11.3 & Vertical & partial \\
\cline { 2 - 5 } & $\mathrm{H}_{23}-\mathrm{H}_{27}$ & 5.0 & Horizontal & . \\
\hline
\end{tabular}

generally shifted or their intensities altered [10]. If $\beta$-CD and SA forms a solid inclusion complex, the non-covalent interactions between them such as hydrophobic interactions, vander Waal's interactions and hydrogen bonding lowers the energy of the included part of SA and reduces the absorption intensities of the corresponding bands. We can see that there are apparent differences between the FTIR spectra of $\beta$-CD, SA and SA: $\beta$-CD solid inclusion complex. The FTIR spectra of SA: $\beta-C D$ and that of $\beta$-CD are alike due to (i) the presence of large number of polar groups such as $\mathrm{O}-\mathrm{H}, \mathrm{C}-\mathrm{O}$, etc., that are responsible for intense absorption bands; (ii) the excess of free (unreacted) $\beta$-CD in the $S A: \beta-C D$ inclusion complex sample. However, the inclusion of SA into the $\beta$-CD cavity is evidently confirmed by the bands at $3299,1501 \mathrm{~cm}^{-1}, 1334$ and $828 \mathrm{~cm}^{-1}$. The IR spectrum of SA examined in $\mathrm{KBr}$ pellet, showed one absorption band at 3299 $\mathrm{cm}^{-1}$ for N-H stretching with benzene. $1501 \mathrm{~cm}^{-1}$ was noted for bending vibration of $\mathrm{N}-\mathrm{H}$ group attached with benzene ring. The characteristic peak of $\mathrm{C}-\mathrm{N}$ stretching vibration in aromatic ring appeared at $1334 \mathrm{~cm}^{-1}$, For $\mathrm{N}-\mathrm{H}$ wagging vibration the peak appeared at $828 \mathrm{~cm}^{-1}$. However, in the IR spectrum of SA: $\beta-C D$ Inclusion complex, (formed by stirring method) absorption band due to the stretching (at $3299 \mathrm{~cm}^{-1}$ ) and bending (at $1501 \mathrm{~cm}$ 1) vibrations of $\mathrm{N}-\mathrm{H}$ disappeared, infers that the N-H of SA was entrapped into the $\beta$-CD cavity in the inclusion complex. The C-N stretching vibration peak at $1334 \mathrm{~cm}^{-1}, \mathrm{~N}-\mathrm{H}$ wagging vibration peak at $828 \mathrm{~cm}^{-1}$ shifted in the inclusion complex, indicating the restriction in the vibration of $\mathrm{N}-\mathrm{H}$ group due to the complete entrapment of $\mathrm{N}-\mathrm{H}$ containing aromatic ring into $\beta$-CD. The aromatic C-H bending vibration at $790 \mathrm{~cm}^{-1}$ and $\mathrm{C}-\mathrm{H}$ out of plane at $895 \mathrm{~cm}^{-1}$ disappeared in the inclusion complex. Stretching vibration of $\mathrm{C}-\mathrm{C}$ in aromatic ring at $1558 \mathrm{~cm}^{-1}$ of SA shifted to $1560 \mathrm{~cm}^{-1}$ in the SA: $\beta$-CD complex. The inclusion complex FTIR spectra peaks are $30-40 \%$ weaker than the free SA molecule. The SA: $\beta$-CD inclusion complex did not show any new peaks, indicating that no chemical bonds were created between SA and $\beta-C D$ in the formed complex which was also confirmed by molecular modeling studies.

\section{Powder X-ray diffraction spectra}

The formation of inclusion complex can be confirmed by $\mathrm{X}$-ray diffractometry $[11,12]$. The Figure 2 shows the powder $X$-ray diffraction spectra of $\beta-C D$, , SA and SA: $\beta$-CD solid complex. The X-ray spectrum of the inclusion complex shown in Figure 2 $\mathrm{b}$ and $\mathrm{c}$ was evidently different from that of $\beta$-CD Figure $2 \mathrm{a}$. The difference between the spectra of $\beta$-CD and inclusion complex is due to the interaction of $\beta$-CD with SA.

\section{Semi empirical quantum mechanical calculations}

The internal diameter of the $\beta$-CD is approximately $6.5 \AA$ and its height is $7.8 \AA$ (Scheme 1). Considering the shape and dimensions of $\beta-C D$, SA cannot be completely embedded in the

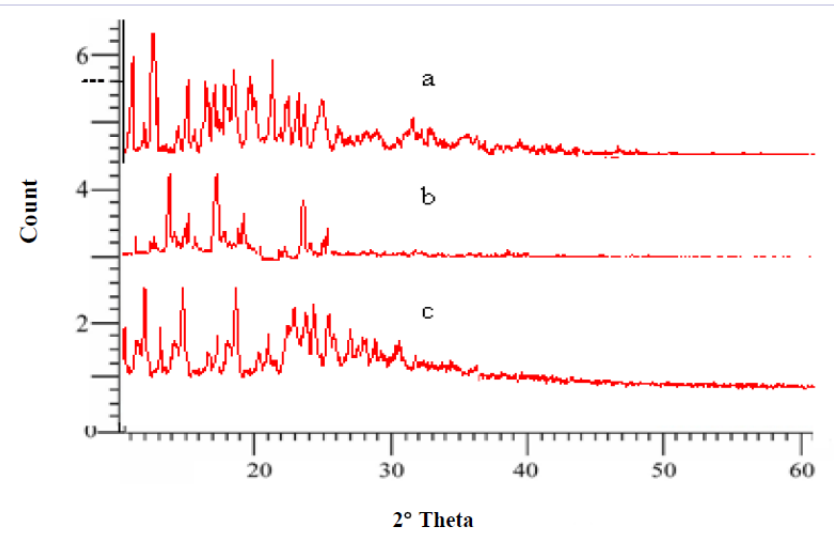

Figure 2: XRD pattern of (a) $\beta$-CD, (b) Salicylanilide and (c) Salicylanilide: $\beta$-CD solid complex. 


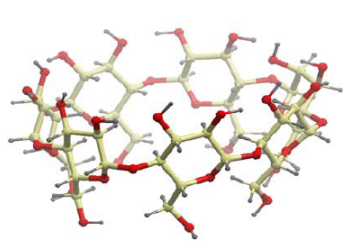

a
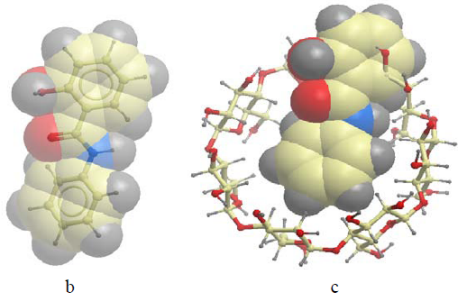

c
Figure 3: Ball and stick representation of (a) $\beta$-CD, (f) SA, and (g) SA: $\beta$ CD 1:1 inclusion complex. oxygen atoms are shown as red balls, carbon atoms as golden balls and sticks, nitrogen atoms as blue balls and hydrogen atoms are shown in grey balls and sticks.

$\beta$-CD cavity. The ground state of SA molecule was optimized using AM1 method. SA the vertical distance between $\mathrm{H}_{17}-\mathrm{H}_{25}$ is $11.3 \AA$ and this is higher than the height of $\beta$-CD. The horizontal distance between $\mathrm{H}_{23}-\mathrm{H}_{27}$ is $5 \AA$ and is less than the internal diameter of $\beta$-CD. Since, the height of SA is higher than that of upper-lower rim of $\beta-\mathrm{CD}$, the $-\mathrm{NH}-\mathrm{CO}-\mathrm{C}_{6} \mathrm{H}_{5}$ aromatic side chain and benzene ring of $\mathrm{SA}$ insertion in the $\beta$-CD cavity is possible as shown in Scheme 1.

\section{Molecular docking study of inclusion process}

The 3D structure of $\beta-C D$ and SA obtained from crystallographic databases are shown in Figure 3. The guest molecule, SA was docked into the cavity of $\beta$-CD using PatchDock server. The PatchDock server gave several possible docked models for the most probable structure based on the energetic parameters; geometric shape complementarity score [13], approximate interface area size and atomic contact energy [14] of the SA: $\beta-C D$ inclusion complex. The docked SA: $\beta-C D$ model (Figure $3 c$ ) with the highest geometric shape complementarity score 3212 approximate interface area size of the complex $381.90 \AA^{2}$ and atomic contact energy $-248.24 \mathrm{kcal} / \mathrm{mol}$ was the highly probable and energetically favourable model.

\section{Conclusions}

In summary, the inclusion complex with 1:1 molar ratio was formed between $\beta$-CD and SA. The $-\mathrm{NH}-\mathrm{CO}-\mathrm{C}_{6} \mathrm{H}_{5}$ aromatic side chain and part of benzene ring of SA was inserted in the $\beta$-CD. The inclusion complex formation of $S A$ with $\beta-C D$, the guest molecules with the $-\mathrm{NH}-\mathrm{CO}-\mathrm{C}_{6} \mathrm{H}_{5}$ aromatic side chain and benzene ring of SA inserted in the $\beta$-CD. Thermodynamic parameter values show the inclusion processes are spontaneous. UV, Fluorescence, XRD, semi empirical and molecular docking results confirms the formation of SA: $\beta$-CD inclusion complex. The inclusion complex formation which was also confirmed by molecular docking studies.

\section{References}

1. Scott Loethen, Jong-Mok Kim, David H. Thompson. Biomedical Applications of Cyclodextrin Based Polyrotaxanes. Polymer Rev. 2007;47(3):383-418.

2. AR Hedges. Industrial Applications of Cyclodextrins. Chem Rev. 1998;98(5):2035-2044. DOI: 10.1021/cr970014w

3. Dina Schneidman-Duhovny, Yuval Inbar, Ruth Nussinov, Haim J Wolfson. PatchDock and SymmDock: servers for rigid and symmetric docking. Nucl Acids Res. 2005;33(suppl 2); 363-367.

4. YH Kim, DW Cho, M Yoon. Observation of Hydrogen-Bonding Effects on Twisted Intramolecular Charge Transfer of $p$ - $(N, N$-Diethylamino $)$ benzoic Acid in Aqueous Cyclodextrin Solutions J Phys Chem. 1996;100(39):15670-15676. DOI: 10.1021/jp9613652

5. YB Jiang J. Photochem. Photobiol. A Chem. 1995;88:109-116.

6. YV Ilichev, W Kuhnle, KA Zachariasse. Intramolecular Charge Transfer in Dual Fluorescent 4-(Dialkylamino) benzonitriles. Reaction Efficiency Enhancement by Increasing the Size of the Amino and Benzonitrile Subunits by Alkyl Substituents. J Phys Chem. 1998;102(28):5670-5680.

7. S Santra, SK Dogra, J Photochem. Photobiol. A Chem. 1996;101:221227.

8. P Bortolos, S Monti. cis .dblharw. trans Photoisomerization of azobenzene-cyclodextrin inclusion complexes. J Phys Chem. 1987;91:(19):5046-5050.

9. HA Benesi, JH Hildebrand. A Spectrophotometric Investigation of the Interaction of Iodine with Aromatic Hydrocarbons. J Am Chem Soc. 1949;71(8):2703-2707.

10.L Szente. Analytical Methods for Cyclodextrins, Cyclodextrin derivatives and Cyclodextrin Complexes, in J. Szejtli, and T Osa, Cyclodextrins, in J Lehn et al., (Editor), Comprehensive Supramolecular Chemistry. Pergamon Press, Oxford. 1996;3:253-278.

11.S Scalia, A Molinari, A Casolari, A Maldotti. Euro J Pharm Sci. 2004;22:241-249.

12.T Pralhad, K Rajendrakumar. Study of freeze-dried quercetincyclodextrin binary systems by DSC, FT-IR, X-ray diffraction and SEM analysis. J Pharm Biomed Anal. 2004;34(2):333-339

13.13. D Duhovny, R Nussinov, HJ Wolfson. Efficient Unbound Docking of Rigid Molecules. Algorithms in Bioinformatics. Lecture Notes in Computer Science. Springer Verlag. 2002;2452:185-200.

14. C. Zhang, G. Vasmatzis, J.L.Cornette, C. DeLisi. Determination of atomic desolvation energies from the structures of crystallized proteins. J Mol Biol. 1997;267(3):707-726. 